Article

\title{
Asymptotic behavior of positive solutions of nonlinear fractional differential equations with Caputo-type Hadamard derivative
}

\author{
Said R. Grace ${ }^{1}$, Shurong Sun ${ }^{2}$ and Zhenlai Han ${ }^{2, *}$ \\ 1 Department of Engineering Mathematics, Faculty of Engineering, Cairo University, Orman, Giza 12221, Egypt.; \\ csaidgrace@yahoo.com \\ 2 School of Mathematical Sciences, University of Jinan, Jinan, Shandong 250022, P R China.; psshrong@163.com (S.S) \\ * Correspondence: hanzhenlai@163.com
}

Received: 9 September 2018; Accepted: 28 December 2018; Published: 8 February 2019.

\begin{abstract}
In this paper we are concerned with the problem of asymptotic integration of positive solutions of higher order fractional differential equations with Caputo-type Hadamard derivative of the form ${ }^{C, H} D_{a}^{r} x(t)=e(t)+f(t, x(t)), a>1$, where $r=n+\alpha-1, \alpha \in(0,1), n \in \mathbb{Z}^{+}$. We shall apply our technique to investigate the oscillatory and asymptotic behavior of all solutions of the integral equation $x(t)=e(t)+\int_{a}^{t}\left(\ln \frac{t}{s}\right)^{r-1} k(t, s) f(s, x(s)) \frac{d s}{s}, a>1, r$ is as above.
\end{abstract}

Keywords: Asymptotic behavior, oscillation, Hadamard derivative, Caputo derivative, fractional differential equations.

MSC: 34E10, 34A34.

\section{Introduction}

Consider the initial value problem

$$
{ }^{C, H} D_{a}^{r} x(t)=e(t)+f(t, x(t)), a>1, \delta^{k} x(a)=b_{k}, b_{k}, k=1,2, \cdots n \text { are real constants, }
$$

and the integral equation

$$
x(t)=e(t)+\int_{a}^{t}\left(\ln \frac{t}{s}\right)^{r-1} k(t, s) f(s, x(s)) \frac{d s}{s}, a>1,
$$

where $r=n+\alpha-1, \alpha \in(0,1), n \in \mathbb{Z}^{+},{ }^{C}, H D_{a}^{r} x(t)$ is the Caputo-type Hadamard modification of fractional derivative of a $C^{n}$ - scalar valued function $x(t)$ defined on the interval $[a, \infty)$, which was recently proposed by Jarad et al. [1].

$$
{ }^{C, H} D_{a}^{r} x(t)={ }^{C, H} J_{a}^{n-r} \delta^{n} x(t), \delta:=t \frac{d}{d t},
$$

where $r=n+\alpha-1, \alpha \in(0,1), n \in \mathbb{Z}^{+}$.

In the sequel we assume that:

1. $e:[a, \infty) \rightarrow R^{+}=(0, \infty)$ is a continuous function;

2. $k:[a, \infty) \times[c, \infty) \rightarrow \mathbb{R}$ is a continuous function, and assume that there exists a continuous function, $b:[a, \infty) \rightarrow \mathbb{R}^{+}$such that $|k(t, s)| \leq b(t)$ for all $t>s>a$;

3. $f:[a, \infty) \times \mathbb{R} \rightarrow \mathbb{R}$ is continuous and assume that there exists a continuous function, $h:[a, \infty) \rightarrow(0, \infty)$ and a real number $\lambda, 0<\lambda \leq 1$ and $\gamma$ is a real number such that $0 \leq x f(t, x) \leq t^{\gamma-1} h(t)|x|^{\lambda+1}$, for $x \neq$ 0 , and $t \geq a$.

We only consider those solutions of equation (1) that are continuable and nontrivial in any neighborhood of $\infty$. Such a solution is said to be oscillatory if there exists a sequence $t_{n} \subseteq[c, \infty) t_{n} \rightarrow \infty$ such that $x\left(t_{n}\right)=0$, and it is nonoscillatory otherwise.

The subject of fractional differential equations has recently evolved as an interesting and popular field of research. In fact, fractional derivatives provide an excellent tool for the description of memory and hereditary 
properties of various materials and processes. Many researchers have found that fractional differential equations play important roles in many research areas, such as physics, chemical technology, population dynamics, biotechnology, and economics [2-14].

However, it has been observed that most of the work on the topic involves either Riemann-Liouville or Caputo-type fractional derivative. Besides these derivatives, Hadamard fractional derivative is another kind of fractional derivatives that was introduced by Hadamard in 1892 [15]. This fractional derivative differs from the other ones in the sense that the kernel of the integral (in the definition of Hadamard derivative) contains logarithmic function of arbitrary exponent. For background material of Hadamard fractional derivative and integral, we refer [4,16-27].

The asymptotic behavior results for fractional differential equations with Caputo-type Hadamard derivative are scarce and it seems that there are no such results for such equations. The main objective of this paper is to establish some new criteria for the asymptotic behavior of all positive solutions of equation (1) as well as the oscillatory and asymptotic behavior of the integral equation (2).

We introduce some notations and definition of fractional calculus $[3,4]$.

Definition 1. For at least $n$-times differentiable function $f:(a,+\infty) \rightarrow \mathbb{R}$ the Caputo-type Hadamard derivative of fractional order $\alpha$ is defined as

$$
{ }^{C, H} D_{a}^{r} f(t)=\frac{1}{\Gamma(n-\alpha)} \int_{a}^{t}\left(\ln \frac{t}{s}\right)^{n-\alpha-1} \delta^{n} f(s) \frac{d s}{s}, n-1<\alpha<n, n=[\alpha],
$$

where $\delta=t \frac{d}{d t}$ and $[\alpha]$ denotes the integer part of the real number $\alpha$.

Definition 2. The Hadamard fractional integral of order $r \in \mathbb{R}^{+}$of function $f(t)$ for all $t>a>0$ is defined by

$$
{ }^{H} D_{a}^{-\alpha} f(t)=\frac{1}{\Gamma(\alpha)} \int_{a}^{t}\left(\ln \frac{t}{s}\right)^{\alpha-1} f(s) \frac{d s}{s},
$$

where $\Gamma(\alpha)$ is the Euler Gamma function.

Definition 3. The Hadamard derivative of order $r \in[n-1, n), n \in \mathbb{Z}^{+}$of a function $f(t)$ is given by

$$
{ }^{H} D_{a}^{\alpha} f(t)=\frac{1}{\Gamma(n-\alpha)}\left(t \frac{d}{d t}\right)^{n} \int_{a}^{t}\left(\ln \frac{t}{s}\right)^{n-\alpha-1} f(s) \frac{d s}{s} .
$$

The corresponding Volterra integral equation can be easily derived using Lemma 2.5 in [1] and it takes the form

$$
x(t)=\sum_{k=0}^{n-1} \frac{b_{k}}{k !}\left(\ln \frac{t}{s}\right)^{k}+\frac{1}{\Gamma(r)} \int_{a}^{t}\left(\ln \frac{t}{s}\right)^{r-1}[e(s)+f(s, x(s))] \frac{d s}{s} .
$$

\section{Asymptotic Behavior of equation (1)}

To obtain our main results of this paper, we need the following two lemmas.

Lemma 4. Let $\beta$, $\gamma$ and $p$ be positive constants such that $[p(\beta-1)+1]>0, p(\gamma-1)+1>0$. Then

$$
\int_{a}^{t}\left(\ln \frac{t}{s}\right)^{p(\beta-1)}\left(\ln \frac{s}{a}\right)^{p(\gamma-1)} \frac{d s}{s}=B\left(\ln \frac{t}{a}\right)^{\theta}, t \geq 0
$$

Where $B:=B[p(\gamma-1)+1, p(\beta-1)+1], B[\xi, \eta]=\int_{0}^{1} s^{\xi-1}(1-s)^{\eta-1} d s,(\xi>0, \eta>0)$ and $\theta=p(\beta+\gamma-2)+$ 1.

Proof. Let $\ln \frac{s}{a}=\tau \ln \frac{t}{a}$. Then $s=a\left(\frac{t}{a}\right)^{\tau}, d s=a\left(\frac{t}{a}\right)^{\tau} \ln \left(\frac{t}{a}\right) d \tau$ and $\frac{d s}{s}=\ln \left(\frac{t}{a}\right) d \tau$. Therefore we obtain

$$
\begin{aligned}
\int_{a}^{t}\left(\ln \frac{t}{s}\right)^{p(\beta-1)}\left(\ln \frac{s}{a}\right)^{p(\gamma-1)} \frac{d s}{s} & =\int_{a}^{1}\left(\ln \left(\frac{t}{a}\left(\frac{t}{a}\right)^{-\tau}\right)\right)^{p(\beta-1)}\left(\tau \ln \left(\frac{t}{a}\right)\right)^{p(\gamma-1)} \ln \left(\frac{t}{a}\right) d \tau \\
& =\left(\ln \frac{t}{a}\right)^{p(\beta+\gamma-2)+1} \int_{0}^{1}(1-\tau)^{p(\beta-1)} \tau^{p(\gamma-1)} d \tau .
\end{aligned}
$$


Lemma 5. [28]. If $X$ and $Y$ are nonnogative, then

$$
X^{\lambda}-(1-\lambda) Y^{\lambda}-\lambda X Y^{\lambda-1} \leq 0,0<\lambda<1,
$$

where equality holds if and only if $X=Y$.

In what follows, we let

$$
g(t)=\frac{\left((1-\lambda) \lambda^{\lambda /(1-\lambda)}\right)}{\Gamma(r)} \int_{t_{1}}^{t}\left(\ln \frac{t}{s}\right)^{r-1}\left(\left(\ln \frac{s}{a}\right)^{\gamma-1} m^{\lambda /(\lambda-1)}(s) h^{1 /(1-\lambda)}(s)\right) \frac{d s}{s}
$$

and $0<\lambda<1, t \geq t_{1}$ for some $t_{1} \geq a$, where $m:[a, \infty) \rightarrow(0, \infty)$ is a continuous function.

Now we give sufficient conditions under which any eventually positive solution $x$ of equation (1) satisfies

$$
x(t)=O\left(\ln \frac{t}{a}\right)^{n-1} \text { as } t \rightarrow \infty .
$$

Theorem 6. Let $0<\lambda<1$ and conditions (1), (2) hold and suppose that $p>1, p(r-1)+1>0, p(\gamma-1)+1>0$, $q=\frac{p}{p-1}, \gamma=(n-r)+\frac{1}{q}$,

$$
\begin{aligned}
& g(t)\left(\ln \frac{t}{a}\right)^{1-n} \text { is bounded on }[a, \infty), g(t) \text { is defined by (6), } \\
& \left(\ln \frac{t}{a}\right)^{1-n} \int_{a}^{t}\left(\ln \frac{t}{s}\right)^{r-1}|e(s)| \frac{d s}{s} \text { is bounded for all } t \geq a,
\end{aligned}
$$

and

$$
\int^{\infty}\left(\ln \frac{s}{a}\right)^{(n-1) q} m^{q}(s) d s<\infty .
$$

If $x$ is positive solution of equation (1), then

$$
\limsup _{t \rightarrow \infty}\left(\ln \frac{t}{a}\right)^{1-n} x(t)<\infty
$$

Proof. Let $x(t)$ be an eventually positive solution of equation (1), say $x(t)>0$ for $t \geq t_{1}$ for some $t_{1} \geq a$. Since equation (1) is equivalent to equation (3), we see that there exists a constant $C_{1}>0$ such that

$$
x(t) \leq C_{1}\left(\ln \frac{t}{a}\right)^{r-1}+\frac{1}{\Gamma(r)} \int_{a}^{t}\left(\ln \frac{t}{s}\right)^{r-1}[e(s)+f(s, x(s))] \frac{d s}{s} .
$$

We let $F(t)=e(t)+f(t, x(t))$. In view of condition (2), we can write

$$
\begin{aligned}
x(t) \leq & C_{1}\left(\ln \frac{t}{a}\right)^{n-1}+\frac{1}{\Gamma(r)} \int_{a}^{t_{1}}\left(\ln \frac{t}{s}\right)^{r-1}|F(s)| \frac{d s}{s}+\frac{1}{\Gamma(r)} \int_{t_{1}}^{t}\left(\ln \frac{t}{s}\right)^{r-1} e(s) \frac{d s}{s} \\
& +\frac{1}{\Gamma(r)} \int_{t_{1}}^{t} \ln \left(\frac{t}{s}\right)^{r-1}\left(\ln \frac{s}{a}\right)^{\gamma-1}\left[h(s) x^{\lambda}(s)-m(s) x(s)\right] \frac{d s}{s} \\
& +\frac{1}{\Gamma(r)} \int_{t_{1}}^{t}\left(\ln \frac{t}{s}\right)^{r-1}\left(\ln \frac{s}{a}\right)^{\gamma-1} m(s) x(s) \frac{d s}{s} .
\end{aligned}
$$

Applying (5) of Lemma 5 to $\left[h(s) x^{\lambda}(s)-m(s) x(s)\right]$ with

$$
X=(h)^{1 / \lambda} x \text { and } Y=\left(\frac{1}{\lambda} m\left(h^{-1 / \lambda}\right)\right)^{1 /(\lambda-1)},
$$

we have

$$
h(s) x^{\lambda}(s)-m(s) x(s) \leq(1-\lambda) \lambda^{\lambda /(1-\lambda)} m^{\lambda /(\lambda-1)}(s) h^{1 /(1-\lambda)}(s)
$$


and so,

$$
\begin{aligned}
x(t) \leq & C_{1}\left(\ln \frac{t}{a}\right)^{n-1}+\frac{1}{\Gamma(r)} \int_{a}^{t_{1}}\left(\ln \frac{t}{s}\right)^{r-1}|F(s)| \frac{d s}{s}+\frac{1}{\Gamma(r)} \int_{t_{1}}^{t}\left(\ln \frac{t}{s}\right)^{r-1}|e(s)| \frac{d s}{s} \\
& +\frac{(1-\lambda) \lambda^{\lambda /(1-\lambda)}}{\Gamma(r)} \int_{t_{1}}^{t} \ln \left(\frac{t}{s}\right)^{r-1}\left(\ln \frac{s}{a}\right)^{r-1}\left[m^{\lambda /(\lambda-1)}(s) h^{1 /(1-\lambda)}(s)\right] \frac{d s}{s} \\
& +\frac{1}{\Gamma(r)} \int_{t_{1}}^{t}\left(\ln \frac{t}{s}\right)^{r-1}\left(\ln \frac{s}{a}\right)^{\gamma-1} m(s) x(s) \frac{d s}{s} .
\end{aligned}
$$

or,

$$
\begin{aligned}
x(t) \leq & C_{1}\left(\ln \frac{t}{a}\right)^{n-1}+\frac{1}{\Gamma(r)} \int_{a}^{t_{1}}\left(\ln \frac{t}{s}\right)^{r-1}|F(s)| \frac{d s}{s}+\frac{1}{\Gamma(r)} \int_{t_{1}}^{t}\left(\ln \frac{t}{s}\right)^{r-1}|e(s)| \frac{d s}{s} \\
& +g(t)+\frac{1}{\Gamma(r)} \int_{t_{1}}^{t}\left(\ln \frac{t}{s}\right)^{r-1}\left(\ln \frac{s}{a}\right)^{\gamma-1} m(s) x(s) \frac{d s}{s} \\
\leq & C\left(\ln \frac{t}{a}\right)^{n-1}+\frac{1}{\Gamma(r)} \int_{t_{1}}^{t}\left(\ln \frac{t}{s}\right)^{r-1}\left(\ln \frac{s}{a}\right)^{\gamma-1} m(s) x(s) \frac{d s}{s},
\end{aligned}
$$

or,

$$
\left(\ln \frac{t}{a}\right)^{n-1} x(t):=z(t) \leq 1+C+\frac{\left(\ln \frac{t}{a}\right)^{1-n}}{\Gamma(r)} \int_{t_{1}}^{t}\left(\ln \frac{t}{s}\right)^{r-1}\left(\ln \frac{s}{a}\right)^{\gamma-1} m(s) x(s) \frac{d s}{s},
$$

where $C$ is the upper bound of the function

$$
\left(\ln \frac{t}{a}\right)^{1-n}\left[\frac{1}{\Gamma(r)}\left(\int_{a}^{t_{1}}\left(\ln \frac{t}{s}\right)^{r-1}|F(s)| \frac{d s}{s}\right)+g(t)+\frac{1}{\Gamma(r)} \int_{t_{1}}^{t}\left(\ln \frac{t}{s}\right)^{r-1}|e(s)| \frac{d s}{s}\right] .
$$

By applying the Holder inequality and Lemma 4, we obtain

$$
\begin{aligned}
\int_{t_{1}}^{t}\left(\ln \frac{t}{s}\right)^{r-1}\left(\ln \frac{t}{a}\right)^{\gamma-1} m(s) x(s) \frac{d s}{s} & \leq\left(\int_{t_{1}}^{t}\left(\ln \frac{t}{s}\right)^{p(r-1)}\left(\ln \frac{t}{a}\right)^{p(\gamma-1)} m(s) x(s) \frac{d s}{s}\right)^{1 / p}\left(\int_{t_{1}}^{t}(m(s))^{q} x^{q}(s) d s\right)^{1 / q} \\
& \leq\left(\int_{0}^{t}\left(\ln \frac{t}{s}\right)^{p(r-1)}\left(\ln \frac{t}{a}\right)^{p(\gamma-1)} \frac{d s}{s}\right)^{1 / p}\left(\int_{t_{1}}^{t}(m(s))^{q} x^{q}(s) d s\right)^{1 / q} \\
& \leq\left(B\left(\ln \frac{t}{a}\right)^{\theta}\right)^{1 / p}\left(\int_{t_{1}}^{t}(m(s))^{q} x^{q}(s) d s\right)^{1 / q}
\end{aligned}
$$

where $B=B[p(\gamma-1)+1, p(r-1)+1]$ and $\theta=p(r+\gamma-2)+1$ and by using $\gamma=(n-r)+\frac{1}{q}$, we have

$$
\frac{1}{\Gamma(r)} \int_{t_{1}}^{t}\left(\ln \frac{t}{s}\right)^{r-1}\left(\ln \frac{s}{a}\right)^{\gamma-1} m(s) x(s) \frac{d s}{s} \leq \frac{B^{1 / p}}{\Gamma(r)}\left(\ln \frac{t}{a}\right)^{n-1}\left(\int_{t_{1}}^{t}(m(s))^{q} x^{q}(s) d s\right)^{1 / q} .
$$

Using (13) in (12), we have

$$
z(t) \leq 1+C+\frac{B^{1 / p}}{\Gamma(r)} \int_{t_{1}}^{t}\left(\ln \frac{s}{a}\right)^{(n-1) q}(m(s) z(s))^{q} d s
$$

Using this inequality and the elementary inequality

$$
(x+y)^{q} \leq 2^{q-1}\left(x^{q}+y^{q}\right), x, y \geq 0 \text { and } q>1,
$$

we obtain from (12)

$$
z^{q}(t) \leq 2^{q-1}\left((1+C)^{q}+\left(\frac{B^{1 / p}}{\Gamma(r)}\right)^{q} \int_{t_{1}}^{t}\left(\ln \frac{s}{a}\right)^{(n-1) q} m^{q}(s) z^{q}(s) d s\right) .
$$

If we denote $u(t)=z^{q}(t)$, i.e. $z(t)=u^{1 / q}(t), P=2^{q-1}\left[(1+C)^{q}\right]$ and $Q=2^{q-1}\left(\frac{B^{1 / p}}{\Gamma(r)}\right)^{q}$ then

$$
u(t) \leq P+Q \int_{t_{1}}^{t}\left(\ln \frac{s}{a}\right)^{(n-1) q} m^{q}(s) u(s) d s, t \geq t_{1} \geq a .
$$


The conclusion follows from Gronwall's inequality and we conclude that

$$
\limsup _{t \rightarrow \infty}\left(\ln \frac{t}{a}\right)^{1-n} x(t)<\infty
$$

Similar to the sublinear case, one can easily prove the following results.

Theorem 7. Let $\lambda=1$ and the hypotheses of Theorem 6 hold with $m(t)=h(t)$ and $g(t)=0$. Then the conclusion of Theorem 6 holds.

Example 1. Let $p>1, r=\alpha$. i.e., $n=1,0<\alpha=1-\frac{1}{2 p}<1, \alpha=\gamma$ and $q=\frac{p}{p-1}$. Clearly,

$$
p(\alpha-1)+1=p(\gamma-1)+1=p\left(1-\frac{1}{2 p}-1\right)+1=\frac{1}{2}>0 \text { and } \theta=p(\alpha+\gamma-2)+1=0 .
$$

Let the functions $a(t)$ and $b(t)$ be as in (i) and (ii) with $b(t)$ be a bounded function and let $f(t, x)=t^{\gamma-1} h(t) x^{\lambda}$, where $0<\lambda<1, h: \mathbb{R}^{+} \rightarrow \mathbb{R}^{+}$is a continuous function with $h(t)=m(t)$,

$$
\int^{\infty} h^{q}(s) d s<\infty \text { and } \limsup _{t \rightarrow \infty}\left(\int_{t_{1}}^{t}\left(\ln \frac{t}{s}\right)^{\alpha-1} s^{\gamma-1} h(s) d s\right)<\infty,
$$

and let $e: \mathbb{R}^{+} \rightarrow \mathbb{R}$ be a continuous function with $\lim _{t \rightarrow \infty} \int_{c}^{t}\left(\ln \frac{t}{s}\right)^{\alpha-1}|e(s)| d s<\infty$.

All conditions of Theorem 6 are satisfied and hence every positive solution $x$ of equation (1) is bounded.

\section{Oscillatory and asymptotic behavior of equation (2)}

In this section, we present the following result on the asymptotic behavior of nonoscillatory solutions of equation (2).

Theorem 8. Let $0<\lambda<1$ and conditions (1), (3) hold and suppose that $p>1, q=\frac{p}{p-1}, \gamma=(n-r)+\frac{1}{q}$, $p(r-1)+1>0, p(\gamma-1)+1>0$, the functions $b(t)$ is bounded, (7) and (9) hold and

$$
\limsup _{t \rightarrow \infty}\left(\ln \frac{t}{a}\right)^{1-n} e(t)<\infty \text { and } \liminf _{t \rightarrow \infty}\left(\ln \frac{t}{a}\right)^{1-n} e(t)>-\infty .
$$

If $x(t)$ is any nonoscillatory solution of equation (2), then

$$
\limsup _{t \rightarrow \infty}\left(\ln \frac{t}{a}\right)^{1-n}|x(t)|<\infty
$$

Proof. Let $x(t)$ be an eventually positive solution of equation (2). We may assume that for $t \geq t_{1}$ for some $t_{1} \geq a$,

$$
\begin{gathered}
x(t) \leq e(t)+\int_{a}^{t_{1}}\left(\frac{t}{s}\right)^{r-1}|k(t, s)| f(s, x(s)) \frac{d s}{s}+\int_{t_{1}}^{t}\left(\frac{t}{s}\right)^{r-1}|k(t, s)| f(s, x(s)) \frac{d s}{s}, \\
x(t) \leq e(t)+b(t) \int_{a}^{t_{1}}\left(\ln \frac{t}{s}\right)^{r-1} f(s, x(s)) \frac{d s}{s}+b(t) \int_{t_{1}}^{t}\left(\frac{t}{s}\right)^{r-1}\left(\ln \frac{s}{a}\right)^{\gamma-1} h(s) x^{\lambda}(s) \frac{d s}{s} .
\end{gathered}
$$

We let $F(t)=f(t, x(t))$. In view of conditions (1) and (3) we may then write

$$
\begin{aligned}
x(t) \leq & e(s)+\int_{a}^{t_{1}}\left(\ln \frac{t}{s}\right)^{r-1}|F(s)| \frac{d s}{s} \\
& +b(t) \int_{t_{1}}^{t}\left(\frac{t}{s}\right)^{r-1}\left(\ln \frac{s}{a}\right)^{\gamma-1} h(s)\left[h(s) x^{\lambda}(s)-m(s) x(s)\right] \frac{d s}{s} \\
& +b(t) \int_{t_{1}}^{t}\left(\frac{t}{s}\right)^{r-1}\left(\ln \frac{s}{a}\right)^{\gamma-1} m(s) x(s) \frac{d s}{s} .
\end{aligned}
$$


Proceeding exactly as in the proof of Theorem 6, we obtain

$$
\begin{aligned}
x(t) \leq & e(s)+b(t) \int_{t_{1}}^{t}\left(\frac{t}{s}\right)^{r-1}|F(s)| \frac{d s}{s} \\
& +\left((1-\lambda) \lambda^{\lambda /(1-\lambda)}\right) b(t) \int_{t_{1}}^{t}\left(\frac{t}{s}\right)^{r-1}\left(\ln \frac{s}{a}\right)^{\gamma-1}\left[m^{\lambda /(\lambda-1)}(s) h^{1 /(1-\lambda)}(s)\right] \frac{d s}{s} \\
& +b(t) \int_{t_{1}}^{t}\left(\frac{t}{s}\right)^{r-1}\left(\ln \frac{s}{a}\right)^{\gamma-1} m(s) x(s) \frac{d s}{s}
\end{aligned}
$$

or,

$$
\begin{gathered}
x(t) \leq e(s)+b(t) \int_{a}^{t_{1}}\left(\frac{t}{s}\right)^{r-1}|F(s)| \frac{d s}{s}+b(t) g(t) \\
+b(t) \int_{t_{1}}^{t}\left(\frac{t}{s}\right)^{r-1}\left(\ln \frac{s}{a}\right)^{\gamma-1} m(s) x(s) \frac{d s}{s} .
\end{gathered}
$$

Applying Holder's inequality and Lemma 4, we obtain

$$
\begin{aligned}
\int_{t_{1}}^{t}\left(\frac{t}{s}\right)^{r-1}\left(\ln \frac{s}{a}\right)^{\gamma-1} m(s) x(s) \frac{d s}{s} & \leq\left(\int_{t_{1}}^{t}\left(\ln \frac{t}{s}\right)^{p(r-1)}\left(\ln \frac{s}{a}\right)^{p(\gamma-1)} \frac{d s}{s}\right)^{1 / p}\left(\int_{t_{1}}^{t} m^{q}(s) x^{q}(s) \frac{d s}{s}\right)^{1 / q} \\
& \leq\left(\int_{a}^{t}\left(\ln \frac{t}{s}\right)^{p(r-1)}\left(\ln \frac{s}{a}\right)^{p(\gamma-1)} \frac{d s}{s}\right)^{1 / p}\left(\int_{t_{1}}^{t} m^{q}(s) x^{q}(s) \frac{d s}{s}\right)^{1 / q} \\
& \leq\left(B\left(\ln \frac{t}{a}\right)^{\theta}\right)^{1 / p}\left(\int_{t_{1}}^{t} m^{q}(s) x^{q}(s) \frac{d s}{s}\right)^{1 / q}
\end{aligned}
$$

where $B=B[p(\gamma-1)+1, p(r-1)+1]$ and $\theta=p(r+\gamma-2)+1$. Using $\gamma=(n-r)+\frac{1}{q}$, we have

$$
b(t) \int_{t_{1}}^{t}\left(\ln \frac{t}{s}\right)^{r-1}\left(\ln \frac{s}{a}\right)^{\gamma-1} m(s) x(s) \frac{d s}{s} \leq B^{1 / p} b(t)\left(\ln \frac{t}{a}\right)^{n-1}\left(\int_{t_{1}}^{t} m^{q}(s) x^{q}(s) \frac{d s}{s}\right)^{1 / q} .
$$

It follows from (18) and (20) that

$$
\left(\ln \frac{t}{a}\right)^{1-n} x(t):=z(t) \leq 1+C+c\left(\int_{t_{1}}^{t}\left(\left(\ln \frac{s}{a}\right)^{n-1} m(s)\right)^{q} z^{q}(s) d s\right)^{1 / q}
$$

where $C$ and $c$ are the upper bound of the functions

$$
\left(\ln \frac{t}{a}\right)^{1-n}\left[|e(s)|+b(t) \int_{a}^{t_{1}}\left(\ln \frac{t}{s}\right)^{r-1}|F(s)| d s+b(t) g(t)\right] \text { and } B^{1 / p} b(t),
$$

respectively. The rest of the proof is similar to that of Theorem 6 and conclude that

$$
\limsup _{t \rightarrow \infty}\left(\ln \frac{t}{a}\right)^{1-n} x(t)<\infty
$$

If $x(t)$ is eventually negative, we can set $y=-x$ to see that $y$ satisfies equation (2) with $e(t)$ be replaced by $-e(t)$ and $f(t, x)$ by $-f(t,-y)$. It follows in a similar manner that

$$
\limsup _{t \rightarrow \infty}\left(\ln \frac{t}{a}\right)^{1-n}(-x(t))<\infty
$$

It follows from (22) and (23) that (16) holds. This completes the proof.

Next, by employing Theorem 6 we present the following oscillation result for equation (2).

Theorem 9. Let $0<\lambda<1$, conditions (1)-(3) hold and suppose that $p>1, q=\frac{p}{p-1}, \gamma=n-r-\frac{1}{q}, p(r-1)+1>0$, and $p(\gamma-1)+1>0$. In addition we assume that the function $b(t)\left(\ln \frac{t}{a}\right)^{n-1}$ is bounded and conditions (7), (9) and (15) hold. If

$$
\limsup _{t \rightarrow \infty} e(t)=\infty \text { and } \liminf _{t \rightarrow \infty} e(t)=-\infty
$$


for all $t_{1} \geq a$, then equation (2) is oscillatory.

Proof. Let $x(t)$ be a nonoscillatory solution of equation (2), say $x(t)>0$ for $t \geq t_{1}$ for some $t_{1} \geq 0$. The proof when $x(t)$ is eventually negative is similar. Proceeding as in the proof of Theorem 7 we arrive at (18). Therefore,

$$
\begin{gathered}
x(t) \leq e(t)+b(t) \int_{a}^{t_{1}}\left(\ln \frac{t}{s}\right)^{r-1}|F(s)| \frac{d s}{s}+b(t) g(t) \\
+b(t) \int_{t_{1}}^{t}\left(\ln \frac{t}{s}\right)^{r-1}\left(\ln \frac{s}{a}\right)^{\gamma-1} m(s) x(s) \frac{d s}{s} .
\end{gathered}
$$

Using (19) in the above inequality we have

$$
\begin{aligned}
x(t) \leq & e(t)+b(t) \int_{a}^{t_{1}}\left(\ln \frac{t}{s}\right)^{r-1}|F(s)| \frac{d s}{s}+b(t) g(t)+B^{1 / p} b(t)\left(\ln \frac{t}{a}\right)^{n-1}\left(\int_{t_{1}}^{t} m^{q}(s) x^{q}(s) \frac{d s}{s}\right)^{1 / q} \\
\leq & e(t)+b(t) \int_{a}^{t_{1}}\left(\ln \frac{t}{s}\right)^{r-1}|F(s)| \frac{d s}{s}+b(t) g(t) \\
& +B^{1 / p} b(t)\left(\ln \frac{t}{a}\right)^{n-1}\left(\int_{t_{1}}^{t}\left(\ln \frac{s}{a}\right)^{(n-1) q} m^{q}(s)\left(\left(\ln \frac{s}{a}\right)^{1-n} x(s)\right)^{q} d s\right)^{1 / q} .
\end{aligned}
$$

Clearly, the conclusion of Theorem 6 holds. This together with (7) and (8) imply that the second integral in this inequality is bounded and hence one can easily see that

$$
x(t) \leq M+e(t)
$$

where $M$ is a positive constant. Finally, taking lim inf in (25) as $t \rightarrow \infty$ and using condition (22) result in a contradiction with the fact that $x(t)$ is eventually positive.

Theorem 10. Let $\lambda=1$ and the hypotheses of Theorems 8 or 9 hold with $m(t)=h(t)$ and $g(t)=0$. Then the conclusions of Theorems 8 and 9 hold.

Example 2. Let $p>1,0<r=2-\frac{1}{p} \in(1,2), q=\frac{p}{p-1}$ and $\gamma=2-r+\frac{1}{q}$. Clearly $p(r-1)+1=p\left(2-\frac{1}{p}-1\right)+1=p>1, p(\gamma-1)+1=p\left(2-\gamma+\frac{1}{q}-1\right)+1>0$ and $\theta=p(r+\gamma-2)+1=p$. Let $f(t, x)=\left(\ln \frac{t}{a}\right)^{\gamma-1} h(t) x^{\lambda}$, where $0<\lambda<1, h: \mathbb{R}^{+} \rightarrow \mathbb{R}^{+}$is a continuous function with $h(t)=m(t)$,

$$
\int^{\infty} h^{q}(s) d s<\infty \text { and } \limsup _{t \rightarrow \infty}\left(\ln \frac{t}{a}\right)^{-1}\left(\int_{t_{1}}^{t}\left(\ln \frac{t}{a}\right)^{r-1} s^{\gamma-1} h(s) d s\right)<\infty,
$$

and let $e: \mathbb{R}^{+} \rightarrow \mathbb{R}$ be a continuous function with

$$
\limsup _{t \rightarrow \infty}\left(\ln \frac{t}{a}\right)^{-1} e(t)<\infty \text { and } \liminf _{t \rightarrow \infty}\left(\ln \frac{t}{a}\right)^{-1} e(t)>-\infty
$$

All conditions of Theorem 8 are satisfied and hence every nonocillatory solution $x(t)$ of equation (2) satisfies (16). In addition, if the function $e(t)$ satisfies condition (24), then equation (2) is oscillatory. We may take $e(t)=(\ln t) \sin t$.

Remark 1. We note that Theorems 8 and 9 are valid when $\frac{\theta}{p}=n-1$. Here, we study the cases when

$$
\text { (I) } \sigma:=\frac{\theta}{p}-n+1>0 \text { or }(I I) \sigma<0 .
$$

Case (I) If $\gamma>(n-r)+\frac{1}{q}$, then $\theta>p(r+\gamma-2)+1$ and so $\sigma>0$. In this case we need to impose the condition that the function $b(t)\left(\ln \frac{t}{a}\right)^{\sigma}$ is bounded and the results are valid. 
Case (II) If $\gamma<(n-r)+\frac{1}{q}$, then $\theta<p(r+\gamma-2)+1$ and so $\sigma<0$. In this case condition (9) may be replaced by

$$
\int^{\infty}\left(\ln \frac{s}{a}\right)^{\sigma q}\left(\ln \frac{s}{a}\right)^{(n-1) q} m^{q}(s) d s<\infty .
$$

and the results remain valid. The details are left to the reader.

\section{General Remarks:}

1. The results of this paper are presented in a form which is essentially new and of higher degree of generality.

2. It would be of interest to study equations (1) and (2) when $f$ satisfies condition (ii) with $\lambda>1$.

\section{Conclusion}

We are concerned with the problem of asymptotic integration of positive solutions of higher order fractional differential equations with Caputo-type Hadamard derivative, and establish some new criteria for the asymptotic behavior of all positive solutions of equation (1) as well as the oscillatory and asymptotic behavior of the integral equation (2).

Author Contributions: All authors contributed equally to the writing of this paper. All authors read and approved the final manuscript.

Conflicts of Interest: "The authors declare no conflict of interest."

\section{References}

[1] Jarad, F., Abdeljawad, T., \& Baleanu, D. (2012). Caputo-type modification of the Hadamard fractional derivatives. Advances in Difference Equations, 2012(1), 142.

[2] Samko, S. G., Kilbas, A. A., \& Marichev, O. I. (1993). Fractional integrals and derivatives: theory and applications. Gordon and Breach Science, Yverdon, Switzerland.

[3] Podlubny, I. (1998). Fractional differential equations: an introduction to fractional derivatives, fractional differential equations, to methods of their solution and some of their applications (Vol. 198). Elsevier.

[4] Kilbas, A. A. A., Srivastava, H. M., \& Trujillo, J. J. (2006). Theory and applications of fractional differential equations (Vol. 204). Elsevier Science Limited.

[5] Baleanu, D. (2012). Fractional calculus: models and numerical methods (Vol. 3). World Scientific.

[6] Agarwal, R. P., Zhou, Y., \& He, Y. (2010). Existence of fractional neutral functional differential equations. Computers \& Mathematics with Applications, 59(3), 1095-1100.

[7] Baleanu, D., Mustafa, O. G., \& Agarwal, R. P. (2011). On $L_{p}$-solutions for a class of sequential fractional differential equations. Applied Mathematics and Computation, 218(5), 2074-2081.

[8] Ahmad, B., \& Nieto, J. J. (2011). Riemann-Liouville fractional integro-differential equations with fractional nonlocal integral boundary conditions. Boundary Value Problems, 2011(1), 36.

[9] Ahmad, B., Ntouyas, S., \& Alsaedi, A. (2011). New Existence Results for Nonlinear Fractional Differential Equations with Three-Point Integral Boundary Conditions. Advances in Difference Equations, 2011.

[10] OŠRegan, D., \& Staněk, S. (2013). Fractional boundary value problems with singularities in space variables. Nonlinear Dynamics, 71(4), 641-652.

[11] Ahmad, B., Ntouyas, S. K., \& Alsaedi, A. (2013). A study of nonlinear fractional differential equations of arbitrary order with Riemann-Liouville type multistrip boundary conditions. Mathematical Problems in Engineering, 2013.

[12] Ahmad, B., \& Nieto, J. J. (2013). Boundary value problems for a class of sequential integrodifferential equations of fractional order. Journal of Function Spaces and Applications, 2013.

[13] Zhang, L., Ahmad, B., Wang, G., \& Agarwal, R. P. (2013). Nonlinear fractional integro-differential equations on unbounded domains in a Banach space. Journal of Computational and Applied Mathematics, 249, 51-56.

[14] Liu, X., Jia, M., \& Ge, W. (2013). Multiple solutions of a $p$-Laplacian model involving a fractional derivative. Advances in Difference Equations, 2013(1), 126.

[15] Hadamard, J. (1892). Essai sur l'etude des fonctions, donnees par leur developpement de Taylor. Journal de Mathematiques pures et appliquées, 8, 101-186.

[16] Butzer, P. L., Kilbas, A. A., \& Trujillo, J. J. (2002). Compositions of Hadamard-type fractional integration operators and the semigroup property. Journal of Mathematical Analysis and Applications, 269(2), 387-400.

[17] Butzer, P. L., Kilbas, A. A., \& Trujillo, J. J. (2002). Fractional calculus in the Mellin setting and Hadamard-type fractional integrals. Journal of Mathematical Analysis and Applications, 269(1), 1-27. 
[18] Butzer, P. L., Kilbas, A. A., \& Trujillo, J. J. (2002). Mellin transform analysis and integration by parts for Hadamard-type fractional integrals. Journal of Mathematical Analysis and Applications, 270(1), 1-15.

[19] Anatoly, A. K. (2001). Hadamard-type fractional calculus. Journal of the Korean Mathematical Society, 38(6), 1191-1204.

[20] Kilbas, A., \& Trujillo, J. (2003). Hadamard-type integrals as G-transforms. Integral Transforms and Special Functions, 14(5), 413-427.

[21] Coffey, W., \& Kalmykov, Y. P. (2012). The Langevin equation: with applications to stochastic problems in physics, chemistry and electrical engineering (Vol. 27). World Scientific.

[22] Mishra, S., Mishra, L. N., Mishra, R. K., \& Patnaik, S. (2019). Some applications of fractional calculus in technological development. Journal of Fractional Calculus and Applications, 10(1), 228-235.

[23] Pathak, H. K. (2013). A study on some problems on existence of solutions for nonlinear functional-integral equations. Acta Mathematica Scientia, 33(5), 1305-1313.

[24] Mishra, L. N., \& Sen, M. (2016). On the concept of existence and local attractivity of solutions for some quadratic Volterra integral equation of fractional order. Applied Mathematics and Computation, 285, 174-183.

[25] Mishra, L. N., Sen, M., \& Mohapatra, R. N. (2017). On existence theorems for some generalized nonlinear functional-integral equations with applications. Filomat, 31(7), 2081-2091.

[26] Mishra, L. N., \& Agarwal, R. P. (2016). On existence theorems for some nonlinear functional-integral equations. Dynamic Systems and Applications, 25(3), 303-320.

[27] Mishra, L. N. (2017). On existence and behavior of solutions to some nonlinear integral equations with Applications. National Institute of Technology, Silchar, 788(010).

[28] Hardy, G. H., Littlewood, J. E., \& Pólya, G. (1959). Inequalities. Cambridge university press.

(c) 2019 by the authors; licensee PSRP, Lahore, Pakistan. This article is an open access article distributed under the terms and conditions of the Creative Commons Attribution (CC-BY) license (http://creativecommons.org/licenses/by/4.0/). 